\title{
Usefulness of combined white blood bell count and blood glucose for predicting in-hospital outcomes in acute ischemic stroke
}

Shoujiang You ${ }^{1}$, Chongke Zhong ${ }^{2}$, Huaping Dư ${ }^{3}$, Yu Zhang ${ }^{1}$, Danni Zheng ${ }^{4}$, Chenhong Qiu ${ }^{5}$, Yongjun Cao ${ }^{1}$, Chun-Feng Liu ${ }^{1}$

1. The Second Affiliated Hospital of Soochow University, Department of Neurology, Suzhou, China.

2. School of Public Health- Medical College of Soochow University, Department of Epidemiology, Suzhou, China.

3. The Affiliated Wujiang Hospital of Nantong University, Department of Neurology, Suzhou, China.

4. University of New South Wales, The George Institute for Global Health, Sydney, Australia.

5. Suzhou Hospital Affiliated to Nanjing Medical University, Department of Neurology, Suzhou, China

\section{Background and aims}

High white blood cell (WBC) count and blood glucose are the risk factors for mortality and pneumonia after acute ischemic stroke (AIS). We investigated the association between combination effect of WBC count and blood glucose upon hospital admission and in-hospital mortality and pneumonia in acute AIS patients.

\section{Methods}

A total of 3,124 AIS patients enrolled from December 2013 to May 2014 across 22 hospitals in Suzhou city were included in the present study. We divided patients into 4 groups according to their level of WBC count and blood glucose level: NWNG (normal WBC count and normal glucose), NWHG (normal WBC count and higher glucose), HWNG (higher WBC count and normal glucose) and HWHG (higher WBC count and higher glucose). Cox proportional hazard and logistic model was used to estimate the effect of combination effect of WBC count and blood glucose on all cause in-hospital mortality and pneumonia in AIS patients.

\section{Resullts}

Patients with HWHG were associated with a 2.89-fold increase in the risk of in-hospital mortality in comparison to NWNG (adjusted hazard ratio [HR] 2.89; 95\% confidence interval [CI], 1.59-5.23; $P$-trend $<0.001)$ after adjusting for potential covariates. The risk of pneumonia is significant higher in patients with HWHG comparison to those with NWNG (adjusted odds ratio [OR] 2.62; 95\% CI, 1.67-4.10; $P$-trend $<0.001)$. The $\mathrm{C}$-statistic of the combination of WBC count and blood glucose were significantly larger than WBC count or blood glucose alone for in-hospital mortality and pneumonia(all $P<0.001$ )

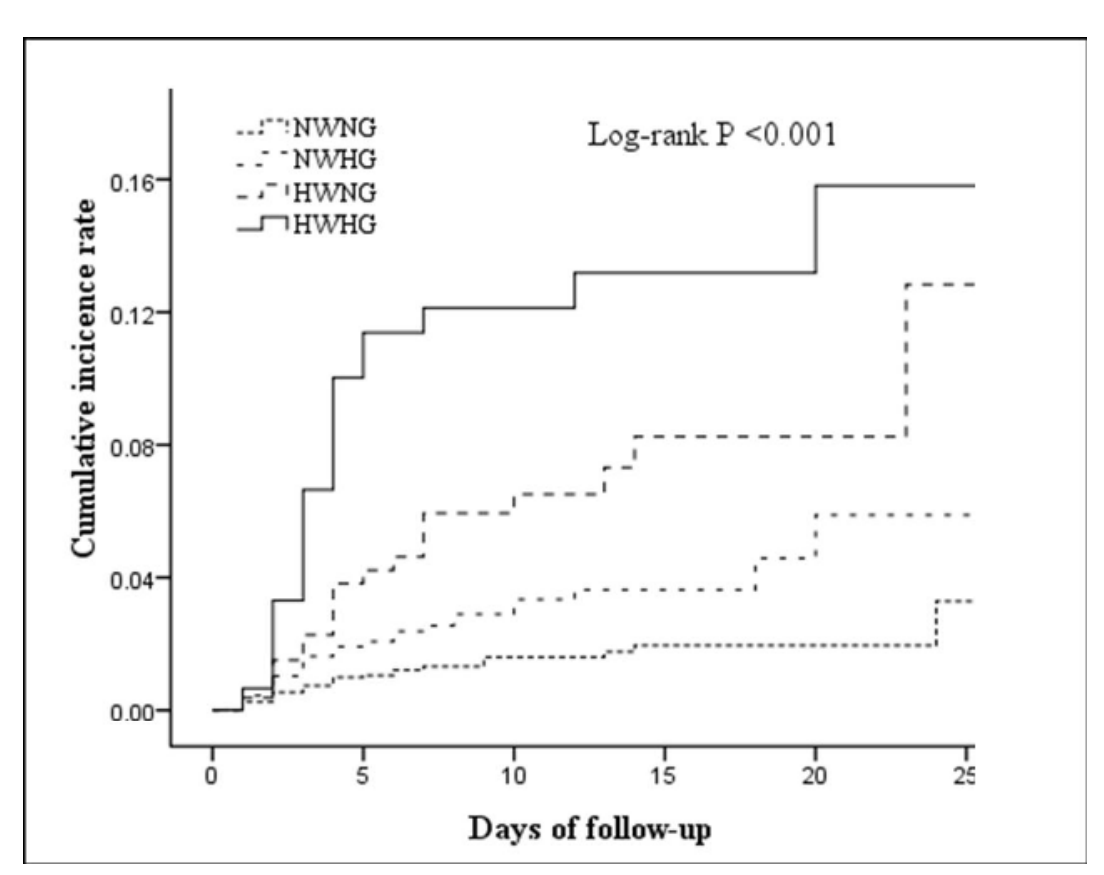

Fig.1Cumulative incidence curves of in-hospital mortality by WBC count and blood glucose level

This work was supported in part by grants from the National Natura Science Foundation of China (81471195), Suzhou Clinical Research Center of Neurological Disease (Szzx201503), the Second Affiliated Hospital of Soochow University Preponderant Clinic Discipline Group Project Funding (XKQ2015002)

\section{Conclusions}

High WBC count with high blood glucose levels at admission was independently associated with in-hospital mortality and pneumonia in AIS patients. Moreover, the combination of WBC count and blood glucose appeared to be a better predictor than WBC count or blood glucose alone.

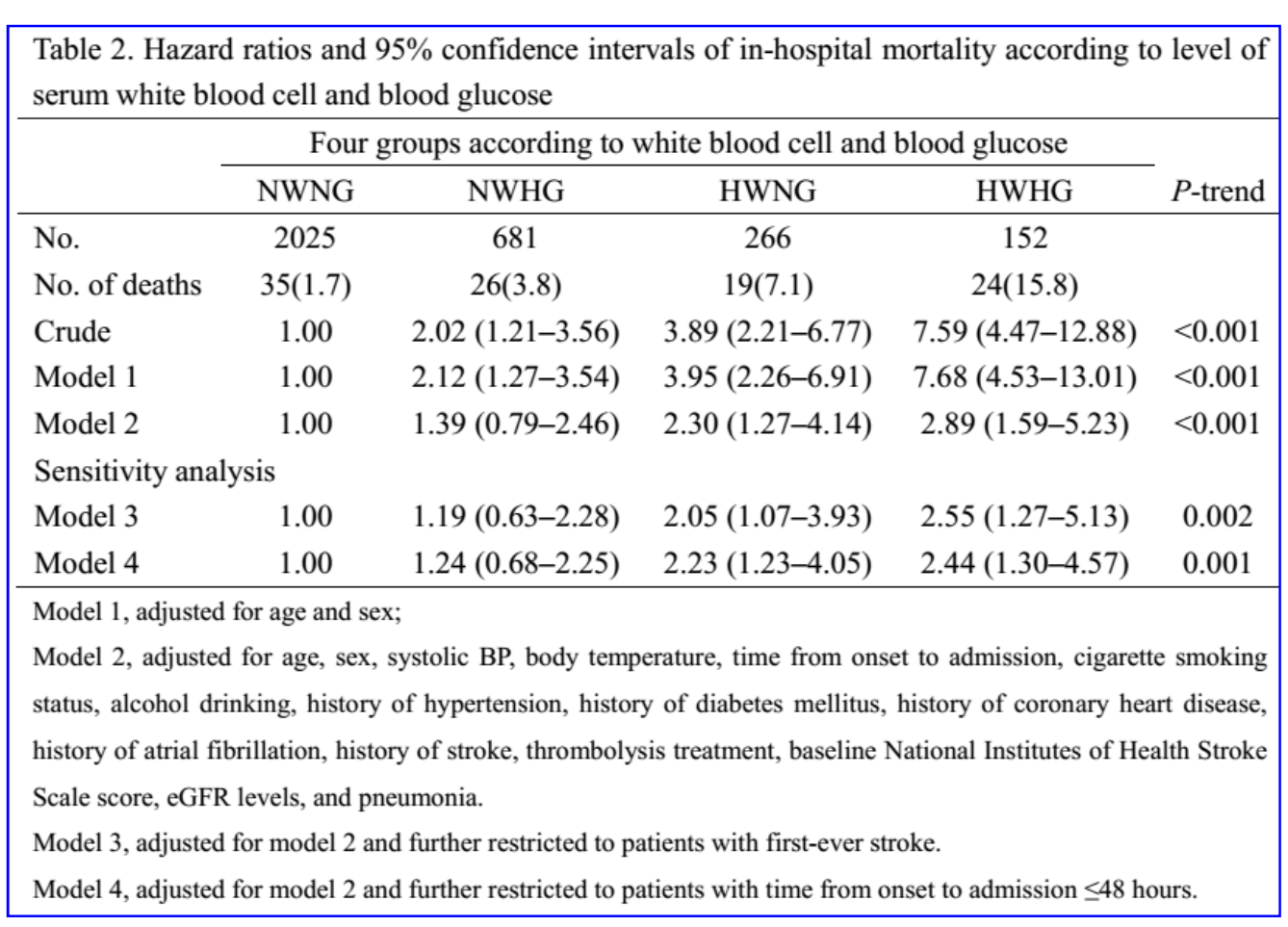

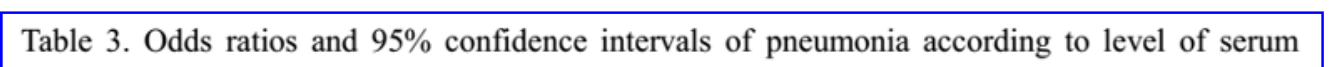
white blood cell and blood glucose

\begin{tabular}{|c|c|c|c|c|c|}
\hline & \multicolumn{4}{|c|}{ Four groups according to white blood cell and blood glucose } & \multirow[b]{2}{*}{$P$-trend } \\
\hline & NWNG & NWHG & HWNG & HWHG & \\
\hline No. & 2025 & 681 & 266 & 152 & \\
\hline No. of deaths & $265(13.1)$ & $125(18.4)$ & $85(32.0)$ & $60(39.5)$ & \\
\hline Crude & 1.00 & $1.49(1.18-1.89)$ & $3.12(2.34-4.16)$ & $4.33(3.05-6.15)$ & $<0.001$ \\
\hline Model 1 & 1.00 & $1.59(1.25-2.02)$ & $3.67(2.70-5.00)$ & $5.26(3.61-7.66)$ & $<0.001$ \\
\hline Model 2 & 1.00 & $1.34(0.98-1.82)$ & $2.06(1.45-2.93)$ & $2.62(1.67-4.10)$ & $<0.001$ \\
\hline \multicolumn{6}{|c|}{ Sensitivity analysis } \\
\hline Model 3 & 1.00 & $1.13(0.78-1.62)$ & $2.17(1.47-3.21)$ & $2.43(1.44-4.10)$ & $<0.001$ \\
\hline Model 4 & 1.00 & $1.25(0.89-1.77)$ & $1.88(1.28-2.77)$ & $2.72(1.68-4.41)$ & $<0.001$ \\
\hline \multicolumn{6}{|c|}{ Model 1, adjusted for age and sex; } \\
\hline \multicolumn{6}{|c|}{$\begin{array}{l}\text { Model 2, adjusted for age, sex, systolic BP, body temperature, time from onset to admission, cigarette smoking } \\
\text { status, alcohol drinking, history of hypertension, history of diabetes mellitus, history of coronary heart disease, } \\
\text { history of atrial fibrillation, history of stroke, thrombolysis treatment, baseline National Institutes of Health Stroke } \\
\text { Scale score, eGFR levels. }\end{array}$} \\
\hline
\end{tabular}
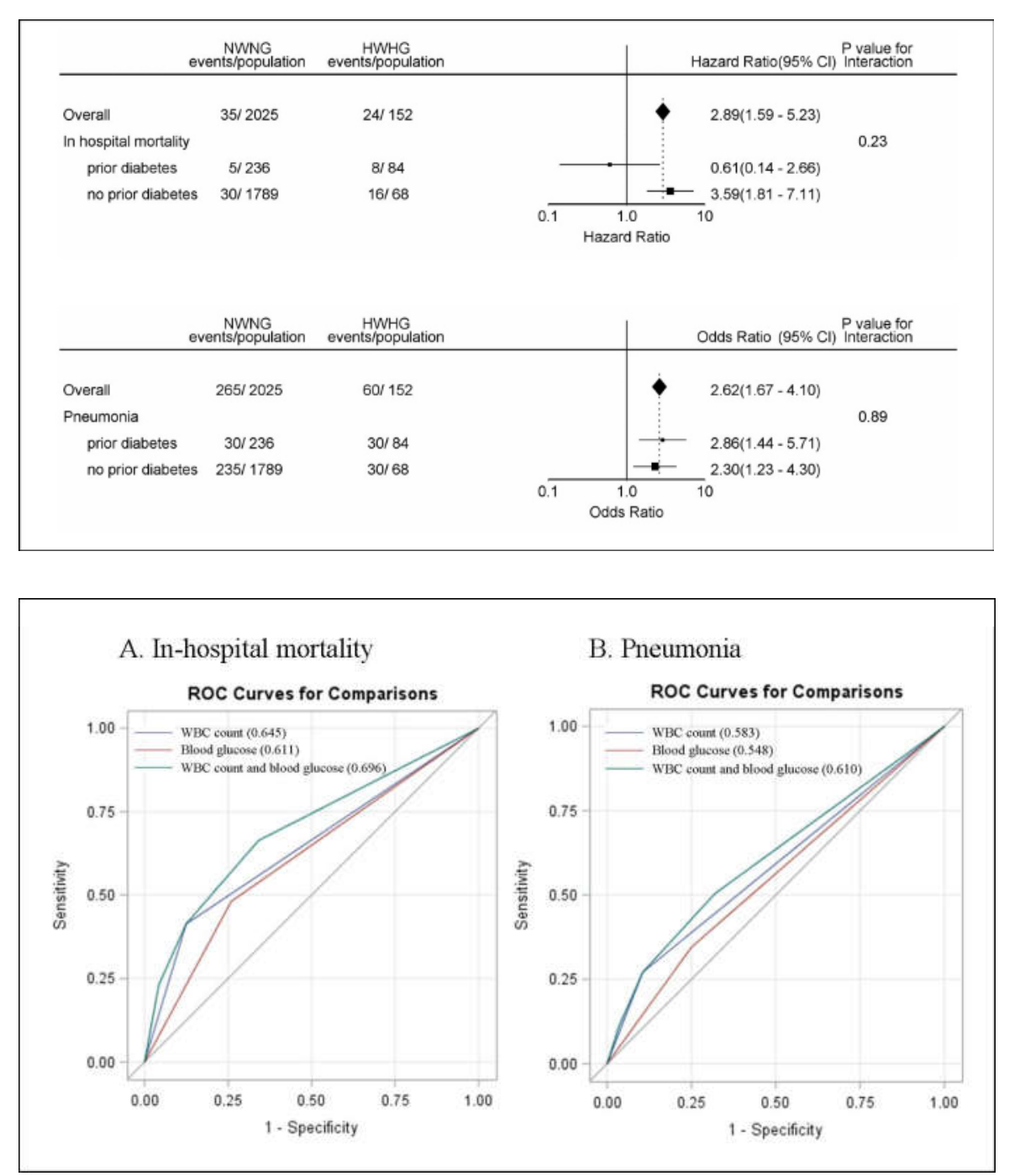

Fig.2 Association between coexisting higher WBC count and higher blood glucose on inhospital mortality and pneumonia in AIS patients

Fig.3 ROC curve of combined WBC count and blood glucose level on in-hospital mortality and pneumonia 